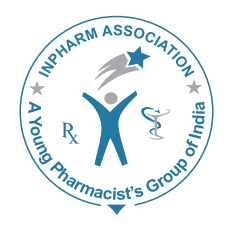

JVP

\title{
Design of Vitamin E d- $\alpha$-Tocopheryl Polyethylene Glycol 1000 Succinate-Emulsified Poly (D,L-Lactide-co-Glycolide) Nanoparticles: Influence of Duration of Ultrasonication Energy
}

\author{
Şengel CT, Hasçiçek C, Gönül N \\ Department of Pharmaceutical Technology, Faculty of Pharmacy, Ankara University, \\ Tandogan, Ankara, Turkey
}

Address for correspondence: Dr. Nurşin Gönül; E-mail: gonul@pharmacy.ankara.edu.tr

\begin{abstract}
The aim of this research was to investigate the effect of the duration of ultrasonication energy on the physicochemical characteristics of the nano-sized particulate drug delivery systems. For this purpose, meloxicam-loaded vitamin $E$ d- $\alpha$-tocopheryl polyethylene glycol 1000 succinate (TPGS)-emulsified poly (D,L-lactide-co-glycolide) (PLGA) nanoparticles were designed by using ultrasonication-solvent evaporation technique and were characterized by photon correlation spectroscopy for size and size distribution, scanning electron microscopy for surface morphology and laser Doppler anemometry for surface charge. Ultraviolet -spectrophotometer was used to measure the drug encapsulation efficiency and to obtain in vitro drug release profile. The results showed that the physicochemical properties of the prepared nanoparticles are effectively controlled by the amount of shear stress transferred from the energy source to the emulsion, which is strongly correlated to the ultrasonication time.
\end{abstract}

Key words: Nanoparticles, PLGA, sonication time, ultrasonication-solvent evaporation technique, vitamin E d- $\alpha$-tocopheryl polyethylene glycol 1000 succinate

\section{INTRODUCTION}

Polymeric nanoparticles are colloidal carrier systems that have been extensively investigated in biomedical and biotechnological areas, especially in drug delivery systems for drug targeting. They could provide a means of modifying the distribution of an active substance

\begin{tabular}{|c|l|}
\hline \multicolumn{2}{|c|}{ Access this article online } \\
\hline Quick Response Code: & \multirow{2}{*}{ Website: } \\
\cline { 1 - 1 } & www.jyoungpharm.in \\
& \\
\hline
\end{tabular}

in vivo and of increasing its concentration in the target tissue, thereby improving efficacy. ${ }^{[1,2]}$ Their capabilities as drug delivery systems with site-specific delivery are dependent on their physicochemical properties, especially particle size, size distribution and surface morphology. Recent research has indicated that the physicochemical characteristics of nanoparticles play important roles in their adhesion to and interaction with the biological cells in the targeted tissues. The physicochemical properties of the nanoparticles were shown to be affected by several formulation and process parameters, such as power and duration of sonication energy applied, drug and polymer concentration, solvent volume, polymer molecular weight, type and concentration of emulsifier, removal rate of organic solvents, aqueous-to-organic 
phase ratio, and process temperature. Among them, sonication parameters, especially sonication time, are the most commonly evaluated factors because of their direct influence on the particle size. ${ }^{[3,4]}$ The diameter of nanoparticles is a crucial parameter determining the extent and rate at which they are removed from the circulation and their biodistribution. Indeed, particle size also affects drug loading and release from the nanoparticulate systems. ${ }^{[5,6]}$

Several reports concerning the manufacture of polymeric nanoparticles have been described within the nano range using different preparation techniques. The solvent evaporation method is one of the fabricating techniques for nanoparticles that can produce nano-sized particles by using ultrasonication or homogenization procedures. ${ }^{[1,4]}$ Ultrasonication and homogenization methods are two variations of the emulsification-solvent evaporation method, and between these two procedures, ultrasonication is the more effective method for producing smaller size nanoparticles. This can be attributed to the capability with ultrasonication of applying higher energy and shear forces during the manufacturing process that strongly break down the emulsion into nanodroplets, which form nanoparticles upon solvent evaporation. In this process, ultrasonic vibrations are transmitted into the emulsion as ultrasonic waves consisting of alternate expansions and compressions, such that an extremely higher energy is released into the emulsion to break down the droplets than is possible with mechanical homogenizers. Furthermore, ultrasonic processing is fast and highly reproducible when compared to mechanical homogenizers. ${ }^{[5,7,8]}$

The primary goal of this research was to design a polymeric nanoparticulate drug delivery system based on a biodegradable polymer, PLGA, and to investigate the effect of sonication time (3, 6 and 9 minutes [min]) on the physicochemical characteristics of the nanoparticles, which were prepared by ultrasonication-solvent evaporation technique. Meloxicam, which is an enolic acid-type non -steroidal anti-inflammatory drug, was used as an ideal hydrophobic molecule for incorporation in nanoparticle systems prepared with this processing method. In this research, vitamin E TPGS, a new water-soluble derivative of natural vitamin $\mathrm{E}$, was selected as an emulsifier in the formulations due to its several advantages, including its bulky structure and large surface area characteristics, which make it an excellent emulsifier. Moreover, it provides a desired drug release profile and can be easily washed away from the nanoparticle surface. ${ }^{[9-11]}$

\section{MATERIALS AND METHODS}

\section{Materials}

Meloxicam was chosen as a model drug (Ulkar Chemical. Co., İstanbul, Turkey). PLGA with a copolymer ratio of D,L - lactide to glycolide of 50:50 (MW: 40.000-75.000 $\mathrm{Da}$ as indicated by the supplier, Sigma-Aldrich Chemical. Co., Munich, Germany) was used as a biodegradable polymer. Vitamin E TPGS was used as an emulsifier in the emulsification process (Eastman Chemical, USA). Methylene chloride (Merck Chemical. Co., Darmstadt, Germany) and purified water (Milli-Q, Millipore Corp., MA, USA) were used as an organic solvent and suspending medium, respectively.

\section{Preparation of nanoparticles}

PLGA nanoparticle formulations were prepared using emulsification by sonication-solvent evaporation method! ${ }^{[9,10]}$ In this technique, $100 \mathrm{mg}$ PLGA and $10 \mathrm{mg}$ meloxicam were dissolved in $4 \mathrm{ml}$ methylene chloride at room temperature. The aqueous phase was prepared by dissolving vitamin $\mathrm{E}$ TPGS in $60 \mathrm{ml}$ Milli-Q water. The organic phase was probesonicated (Bandelin Sonopuls HD 2070, Bandelin Elec., Germany) with the aqueous phase containing vitamin $\mathrm{E}$ TPGS continuously at $55 \mathrm{~W}$ of energy output (amplitude $80 \%$ ), pulse duty $0.7 \mathrm{sec}$ per cycle, in an ice bath for 3 , 6 and 9 min. The organic phase was then removed by evaporation, leaving behind a colloidal suspension of PLGA nanoparticles in water. Nanoparticles were recovered by ultracentrifugation at $30.000 \mathrm{rpm}$ for 1 hour (h) (Optima XL-100K Ultracentrifuge, Beckman Coulter Inc., USA) and washed with Milli-Q water to remove excess vitamin E TPGS. The nanoparticle suspension was then lyophilized (Christ Gamma 2-16 LSC, Martin Christ Gef., Germany) for $48 \mathrm{~h}$ to obtain a dry powder.

\section{Characterization of PLGA nanoparticles}

For the determination of the encapsulation efficiency of the lyophilized nanoparticles, firstly the percentage of entrapped drug (percent drug loading) was estimated. A known amount of nanoparticles was dissolved in methylene chloride (direct method) and ultrasonicated with $40 \%$ power output, pulse duty $0.7 \mathrm{sec}$ per cycle (Bandelin Sonopuls HD 2070, Bandelin Elec., Germany) for $10 \mathrm{~min}$. After eventual dilutions in methylene chloride, the entrapped meloxicam concentration was determined by UV-visible spectrophotometry (Shimadzu UV-1240, Japan) at a wavelength of $342 \mathrm{~nm}(n=6)$. Percent entrapped drug (percent drug loading) was calculated from the ratio of the amount of the drug in nanoparticles to the amount of the 
nanoparticles and multiplying by 100 . Then, the percentage of encapsulation efficiency was estimated from the ratio of percent actual drug entrapped loading to the percent theoretical entrapped drug (drug loading) and multiplying by $100 . .^{[11,12]}$

The mean particle size and size distribution (polydispersity index, PDI) of the nanoparticles were analyzed by photon correlation spectroscopy (PCS). For this analysis, nanoparticles were diluted to the appropriate concentration with Milli-Q water at room temperature. Each analysis was performed at $25^{\circ} \mathrm{C}$ with an angle of detection of $90^{\circ}$.

Surface charge (zeta potential) of the nanoparticles was determined by laser doppler anemometry (LDA). For the determination of the electrophoretic mobility, nanoparticles were diluted with Milli-Q water and placed in the electrophoretic cell.

The PCS and LDA analyses were performed using Zetasizer Nano ZS (Malvern Inst. Inc., Malvern, UK). Each nanoparticle formulation was analyzed five times and mean particle size, PDI and zeta potential were calculated; the average of five measurements was used for analysis.

The shape and surface characteristics of the nanoparticles were observed by scanning electron microscope (SEM) (Jeol JSM-840 A, Japan). A known amount of lyophilized nanoparticles was dusted onto double-sided carbon tape, which was placed on a metallic surface and coated with gold using cold sputter coater (Polaran E 5100) to a thickness of $400 \AA$. The observations were performed at $20 \mathrm{kV}$ electron beam.

\section{In vitro drug release studies}

The in vitro release of meloxicam from vitamin E TPGS emulsified PLGA nanoparticles was determined by dialysis membrane method ${ }^{[13]}$ in phosphate-buffered saline (PBS) $\mathrm{pH} 7.4{ }^{\left[{ }^{[14]}\right.}$ Nanoparticles were suspended in Milli-Q water and transferred into dialysis bags with a molecular mass cutoff of $12.000 \mathrm{Da}$. The bags were placed in a glass container, which was submerged in $100 \mathrm{ml}$ buffer solution and shaken in a water bath (Memmert WB-14, Germany) at $100 \mathrm{rpm}$. The amount of meloxicam released at pre-determined time intervals was determined spectrophotometrically (Shimadzu UV-1240, Japan) at the wavelength of $363 \mathrm{~nm}(n=3)$.

\section{Data analysis}

All data were expressed as mean \pm standard deviation and analyzed by one-way ANOVA followed by the Tukey's post-hoc test with the statistical software package SPSS 9.0 for Windows. Differences at a $P$-value less than 0.05 were considered to be statistically significant and all error bars were presented as standard deviations.

\section{RESULTS AND DISCUSSION}

\section{Preparation of nanoparticles}

In this research, in order to obtain nano-sized particle systems, we used ultrasonication technique. Unlike mechanical homogenizers that rely on pressure and high-velocity motion to shear a fluid into small drops, the ultrasonic probes use only low ultrasonic vibrational energy for atomization, such that an extremely higher energy is released into the emulsion system to form nanosized droplets. With an ultrasonic processor, processing is significantly faster and highly reproducible due to the fact that the energy at the probe tip is high, focused and adjustable. The ultrasonication process was found very suitable for the fabrication of the nano-sized vitamin-E TPGS-emulsified PLGA particles. The production of PLGA nanoparticle formulations by ultrasonicationsolvent evaporation technique related to an investigation on the effect of the various sonication times on the physicochemical characteristics of nanoparticles has been studied earlier, ${ }^{[3-5,7]}$ however, the influence of the duration of sonication energy on the encapsulation efficiency, particle size, size distribution, and release properties of the vitamin E TPGS-emulsified PLGA nanoparticles has not been reported. In this study, it was also evident that vitamin E TPGS was very effective in improving the ultrasonication-emulsification process for the preparation technique of nanoparticles when it was used as an emulsifier in the nanoparticle formulations.

\section{Characterization of nanoparticles}

The results of the physicochemical characterization of the nanoparticles developed are shown in Table 1. The encapsulation efficiency of meloxicam within the vitamin E TPGS-emulsified PLGA nanoparticles was not very high for all nanoparticle formulations and was not affected significantly by the studied durations of ultrasonication energy $(P>0.05)$. The emulsification agent used in the nanoparticle formulations is one of the important factors that influence the drug encapsulation efficiency in the nanoparticles. Our encapsulation efficiency results indicated that vitamin E TPGS is a good but not very effective emulsifier for providing very high drug encapsulation in the nanoparticle formulations containing a hydrophobic drug such as 
meloxicam. It was thought that this result might be an effect of the physicochemical properties (e.g. partition coefficient, aqueous solubility, molecular weight) of the hydrophobic drug used in the nanoparticle formulation and the interaction between meloxicam and vitamin E TPGS during the emulsification process. Similar results were reported by $\mathrm{Mu}$ and Feng about the encapsulation efficiency of the vitamin E TPGSemulsified nanoparticles. ${ }^{[10]}$

The nanoparticle size and size distribution, which are explained with PDI and surface charge, play an important role in determining the mechanism and efficiency of nanoparticle absorption in the targeted area. ${ }^{[7]}$ PDI was an indication of the particle size distribution and referred to the $\log$ normal distribution width of the particle diameter. ${ }^{[11]}$ In order to obtain emulsified systems with the ultrasonication process, the addition of energy is a fundamental step. To verify the influence of this factor on the nanoparticle size and size distribution, ultrasonication time was varied as 3,6 and $9 \mathrm{~min}$. The effect of the ultrasonication time on the particle size and size distribution of the nanoparticles is shown in Table 1. The particle size analysis of the vitamin E TPGS-emulsified nanoparticles indicated that an increase in the sonication time leads to a significant reduction in the nanoparticle size $(P<0.05)$. The increasing duration of sonication increases the energy causing droplet breakdown, thereby increasing the shear stress and resulting in a decrease in particle size. The basic scientific principle governing the size of nanodroplets is that the external energy released from the ultrasonication process provides shear stresses to the emulsion system, which results in the formation of nanodroplets. The size of the droplets is correlated with the magnitude of shear stresses. Any changes in the processing or material parameters that decrease shear stresses will increase the nanodroplet size. The most direct influence on the shear stresses in the emulsion system is exercised by the energy density, which is described by the external energy applied per unit to the total volume of the emulsion. Increasing the energy density directly increases the shear stresses and results in a more efficient droplet breakdown and hence a reduction in nanodroplet size. ${ }^{[3]}$ With the longest time of ultrasonication ( 9 min), a higher energy was released in the emulsification process, which led to a rapid dispersion of the polymeric organic phase as nanodroplets with small size. ${ }^{[4]}$ When the PDI results were investigated based on variation of the sonication time for the formulations prepared, it was observed that all the nanoparticles produced with vitamin E TPGS showed bimodal size distribution, with the PDIs between 0.449 and 0.651 . Another important factor observed was that an increase in sonication time leads to an increase in the production of smaller particles with narrower size distribution. The emulsification can be considered one of the most important steps of the process because an insufficient dispersion of phases results in larger particles with a wide size distribution. Our results were consistent with the reports of the other research groups. ${ }^{[4,5]}$

Zeta potential is an important parameter for the stability of the emulsified systems. Suitable absolute value of zeta potential indicates the presence of suitable electric charge on the surface of the drug-loaded nanoparticles, which can cause strong repellent forces among nano-sized particles to prevent aggregation of the nanoparticles in the dispersion medium..$^{[9,1]}$ The zeta potential values of the nanoparticles indicated that the surface charge was not affected significantly by the variation in the sonication times investigated $P>0.05$ ), as shown in Table 1 . Vitamin E TPGS-emulsified PLGA nanoparticles were found to be stable in dispersion state, possessing suitable absolute values of zeta potential and having negative surface charges.

The shape and surface morphology of the prepared nanoparticles was investigated using SEM. SEM images of the prepared nanoparticles are presented in Figure 1. It seemed that vitamin E TPGS-emulsified PLGA nanoparticles exhibited very spherical shapes within a wide size distribution.

Drug release studies were performed using dialysis method. In vitro release profiles of meloxicam from vitamin E TPGS-emulsified PLGA nanoparticle formulations under in vitro sink conditions are given in Figure 2 . The dissolution data were evaluated statistically

Table 1: Influence of sonication time on the physicochemical characteristics of nanoparticles (mean \pm S.D.)

\begin{tabular}{|c|c|c|c|c|}
\hline $\begin{array}{l}\text { Sonication time of } \\
\text { nanoemulsion ( } \mathrm{min})\end{array}$ & Encapsulation efficiency (\%) & Particle size (nm) (\%) & PDI & $\begin{array}{c}\text { Zeta potential } \\
(\mathrm{mV})\end{array}$ \\
\hline 3 & $57.30 \pm 10.30$ & $474.50 \pm 24.09(71.80) 82.28 \pm 11.68(28.20)$ & $0.651 \pm 0.15$ & $-29.90 \pm 3.27$ \\
\hline 6 & $53.77 \pm 4.67$ & $397.20 \pm 21.10(63.70) 72.25 \pm 15.37(36.30)$ & $0.571 \pm 0.08$ & $-25.20 \pm 4.18$ \\
\hline 9 & $50.91 \pm 0.49$ & $367.40 \pm 26.05(41.00) 68.40 \pm 27.27(59.00)$ & $0.449 \pm 0.06$ & $-20.10 \pm 2.54$ \\
\hline
\end{tabular}

S.D.: Standard deviation, PDI: Polydispersity index 


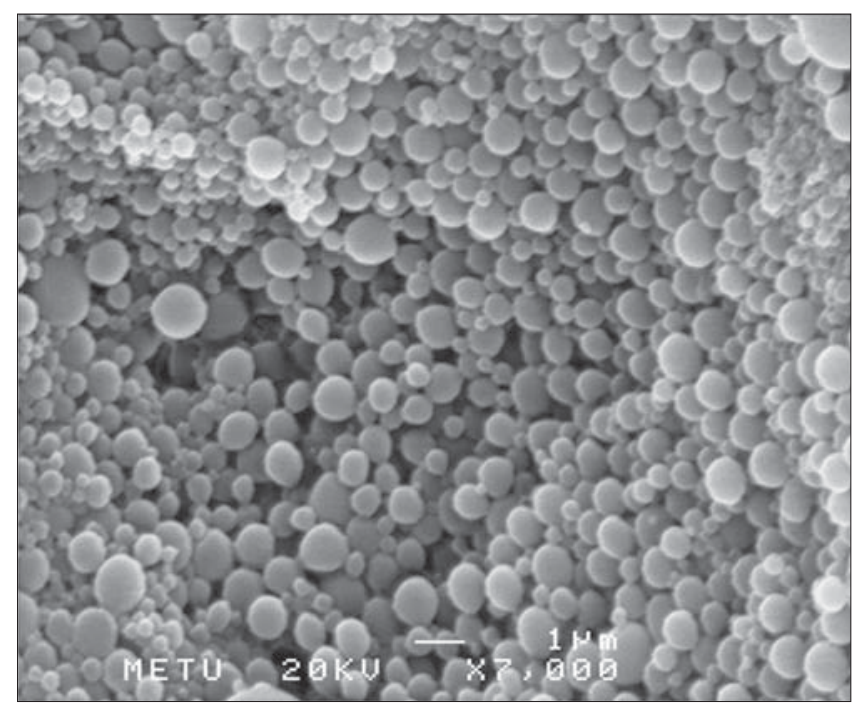

Figure 1: Scanning electron micrographs of vitamin E TPGSemulsified PLGA nanoparticles

by one-way ANOVA with Tukey's post-hoc test and were found to be significantly different depending on the variation in the sonication time for the formulations investigated $(P<0.05)$. Lower meloxicam releases were obtained in the nanoparticles prepared with shorter sonication time. As the sonication time was increased in the emulsification process, the highest energy was released to the emulsion, leading to the formation of smaller nanodroplets, which was directly related to the drug release rate from the nanoparticles. Decreasing the sonication time resulted in an increase in the particle size and thus led to a decrease in the dissolution rate of the drug entrapped in the nanoparticles. From the drug release profiles of the meloxicam-loaded vitamin $\mathrm{E}$ TPGS-emulsified PLGA nanoparticles, it also seemed that the drug release exhibited a biphasic profile with an initial burst effect followed by a sustained release. This finding is in agreement with a report of Zhang and Feng. ${ }^{[9]}$

\section{CONCLUSION}

In the present research, the influence of the sonication time on the physicochemical characteristics of vitamin $\mathrm{E}$ TPGS-emulsified PLGA nanoparticles was systematically assessed. Ultrasonication-solvent evaporation technique was successfully applied for the preparation of vitamin $\mathrm{E}$ TPGS-emulsified PLGA nanoparticles encapsulating meloxicam. It was concluded that among the various formulation parameters, sonication time played an important role in the design of PLGA nanoparticles, and the size of the nanoparticles decreased with an increase in sonication time. The release rate of the entrapped drug can be modified by variation of the sonication time.

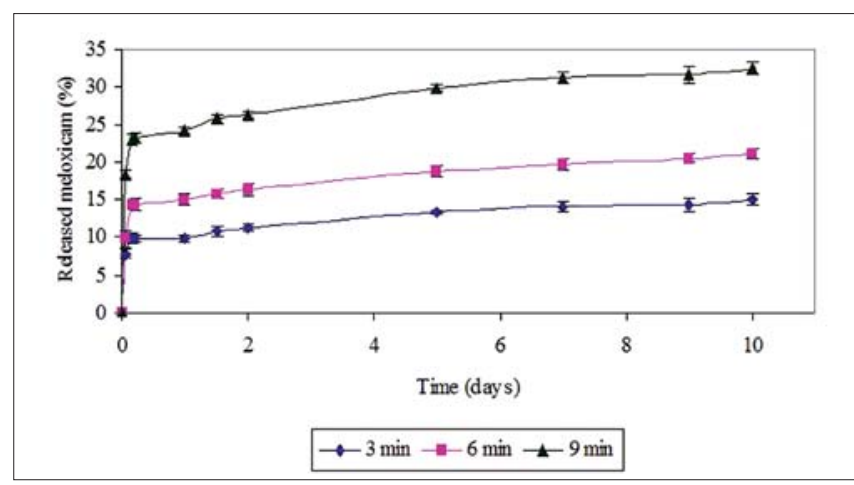

Figure 2: Effect of sonication time (3,6 and $9 \mathrm{~min}$ ) on drug release profiles of vitamin E TPGS-emulsified PLGA nanoparticle formulations over 10 days

\section{REFERENCES}

1. Legrand P, Lesieur S, Bochot A, Gref R, Raatjes W, Barratt G, et al. Influence of polymer behaviour in organic solution on the production of polylactide nanoparticles by nanoprecipitation. Int J Pharm 2007;344:33-43.

2. Zvonar A, Kristl J, Kerc J, Grabnar PA. High celecoxib-loaded nanoparticles prepared by a vibrating nozzle device. J Microencapsul 2009;26:748-59.

3. Budhian A, Siegel SJ, Winey KI. Haloperidol-loaded PLGA nanoparticles: systematic study of particle size and drug content. Int J Pharm 2007;336:367-75.

4. Mainardes RM, Evangelista RC. PLGA nanoparticles containing praziquantel: Effect of formulation variables on size distribution. Int $\mathrm{J}$ Pharm 2005;290:137-44.

5. Song X, Zhao Y, Wu W, Bi Y, Cai Z, Chen Q, et al. PLGA nanoparticles simultaneously loaded with vincristine sulfate and verapamil hydrochloride: systematic study of particle size and drug entrapment efficiency. Int J Pharm 2008;350:320-9.

6. Oeffinger BE, Wheatley MA. Development and characterization of a nanoscale contrast agent. Ultrasonics 2004;42:343-7.

7. Cun D, Foged C, Yang M, Frøkjaer S, Nielsen HM. Preparation and characterization of poly (D,L -lactide - co -glycolide) nanoparticles for siRNA delivery. Int J Pharm 2010;390:70-5.

8. Mccarron P, Donnelly RF, Marouf W. Celecoxib - loaded poly (D,L-lactide -co -glycolide) nanoparticles prepared using a novel and controllable combination of diffusion and emulsification steps as part of the salting-out procedure. J Microencapsul 2006;23:480-98.

9. Zhang Z, Feng SS. Nanoparticles of poly (lactide)/vitamin E TPGS copolymer for cancer chemotherapy: Synthesis, formulation, characterization and in vitro drug release. Biomaterials 2006;27:262-70.

10. Mu L, Feng SS. A novel controlled release formulation for the anticancer drug paclitaxel $\left(\right.$ Taxol $\left.^{\circledR}\right)$ : PLGA nanoparticles containing vitamin E TPGS. J Control Release 2003;86:33-48.

11. Zhang Z, Feng SS. The drug encapsulation efficiency, in vitro drug release, cellular uptake and cytotoxicity of paclitaxel-loaded poly (lactide)tocopheryl polyethylene glycol succinate nanoparticles. Biomaterials 2006;27:4025-33.

12. Şengel-Türk CT, Hasçiçek C, Gönül N. Microsphere-based once-daily modified release matrix tablets for oral administration in angina pectoris. J Microencapsul 2008;25:257-66.

13. Sezgin Z, Yüksel N, Baykara T. Investigation of pluronic and PEG-PE micelles as carriers of mesa-tetraphenyl porphine for oral administration. Int J Pharm 2007;332:161-7.

14. Druckerei CH. European Pharmacopoeia 5.7. Nördlingen: Beck Press; 2007. p. 4932-7.

How to cite this article: Sengel CT, Hasçiçek C, Gönül N. Design of vitamin $\mathrm{E}$ d-a-tocopheryl polyethylene glycol 1000 succinate-emulsified poly (D,L-Lactide-co-glycolide) nanoparticles: Influence of duration of ultrasonication energy. J Young Pharmacists 2011;3:171-5.

Source of Support: Nil, Conflict of Interest: None declared. 